\title{
Existence and Asymptotic Behavior of Boundary Blow-Up Solutions for Weighted $p(x)$-Laplacian Equations with Exponential Nonlinearities
}

\author{
Li Yin, ${ }^{1}$ Yunrui Guo, ${ }^{2}$ Jing Yang, ${ }^{1}$ Bibo Lu, ${ }^{3}$ and Qihu Zhang ${ }^{1,4}$ \\ ${ }^{1}$ Department of Mathematics and Information Science, Zhengzhou University of Light Industry, \\ Zhengzhou, Henan 450002, China \\ ${ }^{2}$ Department of Mathematics, Henan Institute of Science and Technology, Xinxiang, Henan 453003, China \\ ${ }^{3}$ School of Computer Science and Technology, Henan Polytechnic University, Jiaozuo, Henan 454000, China \\ ${ }^{4}$ School of Mathematics and Statistics, Huazhong Normal University, Wuhan, Hubei 430079, China
}

Correspondence should be addressed to Qihu Zhang, zhangqh1999@yahoo.com.cn

Received 21 March 2010; Accepted 3 October 2010

Academic Editor: Pavel Drábek

Copyright (C) $2010 \mathrm{Li}$ Yin et al. This is an open access article distributed under the Creative Commons Attribution License, which permits unrestricted use, distribution, and reproduction in any medium, provided the original work is properly cited.

This paper investigates the following $p(x)$-Laplacian equations with exponential nonlinearities: $-\Delta_{p(x)} u+\rho(x) e^{f(x, u)}=0$ in $\Omega, u(x) \rightarrow+\infty$ as $d(x, \partial \Omega) \rightarrow 0$, where $-\Delta_{p(x)} u=-\operatorname{div}\left(|\nabla u|^{p(x)-2} \nabla u\right)$ is called $p(x)$-Laplacian, $\rho(x) \in C(\Omega)$. The asymptotic behavior of boundary blow-up solutions is discussed, and the existence of boundary blow-up solutions is given.

\section{Introduction}

The study of differential equations and variational problems with nonstandard $p(x)$-growth conditions is a new and interesting topic. On the background of this class of problems, we refer to [1-3]. Many results have been obtained on this kind of problems, for example, [4-18]. On the regularity of weak solutions for differential equations with nonstandard $p(x)$-growth conditions, we refer to $[4,5,8]$. On the existence of solutions for $p(x)$-Laplacian equation Dirichlet problems in bounded domain, we refer to $[7,9,15,18]$. In this paper, we consider the following $p(x)$-Laplacian equations with exponential nonlinearities

$$
\begin{aligned}
& -\Delta_{p(x)} u+\rho(x) e^{f(x, u)}=0, \quad \text { in } \Omega, \\
& u(x) \longrightarrow+\infty, \quad \text { as } d(x, \partial \Omega) \longrightarrow 0,
\end{aligned}
$$


where $-\Delta_{p(x)} u=-\operatorname{div}\left(|\nabla u|^{p(x)-2} \nabla u\right)$ and $\Omega=B(0, R) \subset \mathbb{R}^{N}$ is a bounded radial domain $\left(B(0, R)=\left\{x \in \mathbb{R}^{N}|| x \mid<R\right\}\right)$. Our aim is to give the asymptotic behavior and the existence of boundary blow-up solutions for problem $(\mathrm{P})$.

Throughout the paper, we assume that $p(x), \rho(x)$, and $f(x, u)$ satisfy the following. $\left(\mathrm{H}_{1}\right) p(x) \in C^{1}(\bar{\Omega})$ is radial and satisfies

$$
1<p^{-} \leq p^{+}<+\infty, \quad \text { where } p^{-}=\inf _{\Omega} p(x), \quad p^{+}=\sup _{\Omega} p(x) .
$$
$x \in \Omega$.

$\left(\mathrm{H}_{2}\right) f(x, u)$ is radial with respect to $x, f(x, \cdot)$ is increasing, and $f(x, 0)=0$ for any $\left(\mathrm{H}_{3}\right) f: \Omega \times \mathbb{R} \rightarrow \mathbb{R}$ is continuous and satisfies

$$
|f(x, t)| \leq C_{1}+C_{2}|t|^{\gamma(x)}, \quad \forall(x, t) \in \Omega \times \mathbb{R},
$$

where $C_{1}, C_{2}$ are positive constants and $0 \leq \gamma \in C(\bar{\Omega})$.

$\left(\mathrm{H}_{4}\right) \rho(x) \in C(\Omega)$ is a radial nonnegative function, and there exists a constant $\sigma \in$ $[R / 2, R)$ such that

$$
\rho_{0}(R-r)^{-\beta(r)} \leq \rho(r) \leq \rho_{1}(R-r)^{-\beta_{1}(r)} \text { for } r \in[\sigma, R) \text { uniformly, }
$$

where $\rho_{0}$ and $\rho_{1}$ are positive constants and $\beta(r)$ and $\beta_{1}(r)$ are Lipschitz continuous on $[\sigma, R]$, which satisfy $\beta(r) \leq \beta_{1}(r)<p(r)$ for any $r \in[\sigma, R]$.

The operator $-\Delta_{p(x)} u=-\operatorname{div}\left(|\nabla u|^{p(x)-2} \nabla u\right)$ is called $p(x)$-Laplacian. Specifically, if $p(x) \equiv p$ (a constant), (P) is the well-known $p$-Laplacian problem. If $f(x, u)$ can be represented as $h(x) f(u)$, on the boundary blow-up solutions for the following $p$-Laplacian equations ( $p$ is a constant):

$$
-\Delta_{p} u+h(x) f(u)=0, \quad \text { in } \Omega,
$$

we refer to [19-26], and the following generalized Keller-Osserman condition is crucial

$$
\int_{1}^{\infty} \frac{1}{(F(t))^{1 / p}} d t<+\infty, \quad \text { where } F(t)=\int_{0}^{t} f(s) d s
$$

but the typical form of $p(x)$-Laplacian equation is

$$
-\Delta_{p(x)} u+|u|^{q(x)-2} u=0, \quad \text { in } \Omega,
$$

and there are some differences between the results of (1.4) and (1.6) (see [16]).

On the boundary blow-up solutions for the following $p$-Laplacian equations with exponential nonlinearities ( $p$ is a constant):

$$
-\Delta_{p} u+e^{h(x) f(u)}=0, \quad \text { in } \Omega,
$$


we refer to [20-22], but the results on the boundary blow-up solutions for $p(x)$-Laplacian equations are rare (see [16]).

In [16], the present author discussed the existence and asymptotic behavior of boundary blow-up solutions for the following $p(x)$-Laplacian equations:

$$
\begin{gathered}
-\Delta_{p(x)} u+f(x, u)=0, \quad \text { in } \Omega, \\
u(x) \longrightarrow+\infty, \quad \text { as } d(x, \partial \Omega) \longrightarrow 0,
\end{gathered}
$$

on the condition that $f(x, \cdot)$ satisfies polynomial growth condition.

If $p(x)$ is a function, the typical form of $(\mathrm{P})$ is the following:

$$
-\Delta_{p(x)} u+\rho(x) e^{|u|^{q(x)-2} u}=0
$$

and the method to construct subsolution and supersolution in [16] cannot give the exact asymptotic behavior of solutions for $(\mathrm{P})$. Our results partially generalized the results of [2022].

Because of the nonhomogeneity of $p(x)$-Laplacian, $p(x)$-Laplacian problems are more complicated than those of $p$-Laplacian ones (see [10]); another difficulty of this paper is that $f(x, u)$ cannot be represented as $h(x) f(u)$.

\section{Preliminary}

In order to deal with $p(x)$-Laplacian problems, we need some theories on the spaces $L^{p(x)}(\Omega)$, $W^{1, p(x)}(\Omega)$ and properties of $p(x)$-Laplacian, which we will use later (see $[6,11]$ ). Let

$L^{p(x)}(\Omega)=\left\{u \mid u\right.$ is a measurable real-valued function, $\left.\int_{\Omega}|u(x)|^{p(x)} d x<\infty\right\}$.

We can introduce the norm on $L^{p(x)}(\Omega)$ by

$$
|u|_{p(x)}=\inf \left\{\lambda>\left.0\left|\int_{\Omega}\right| \frac{u(x)}{\lambda}\right|^{p(x)} d x \leq 1\right\} .
$$

The space $\left(L^{p(x)}(\Omega),|\cdot|_{p(x)}\right)$ becomes a Banach space. We call it generalized Lebesgue space. The space $\left(L^{p(x)}(\Omega),|\cdot|_{p(x)}\right)$ is a separable, reflexive, and uniform convex Banach space (see [6, Theorems 1.10,1.14]).

The space $W^{1, p(x)}(\Omega)$ is defined by

$$
W^{1, p(x)}(\Omega)=\left\{u \in L^{p(x)}(\Omega)|| \nabla u \mid \in L^{p(x)}(\Omega)\right\},
$$


and it can be equipped with the norm

$$
\|u\|=|u|_{p(x)}+|\nabla u|_{p(x)}, \quad \forall u \in W^{1, p(x)}(\Omega)
$$

$W_{0}^{1, p(x)}(\Omega)$ is the closure of $C_{0}^{\infty}(\Omega)$ in $W^{1, p(x)}(\Omega) . W^{1, p(x)}(\Omega)$ and $W_{0}^{1, p(x)}(\Omega)$ are separable, reflexive, and uniform convex Banach spaces (see [6, Theorem 2.1]).

If $u \in W_{\text {loc }}^{1, p(x)}(\Omega) \cap C(\Omega), u$ is called a blow-up solution of $(\mathrm{P})$ when it satisfies

$$
\int_{Q}|\nabla u|^{p(x)-2} \nabla u \nabla q d x+\int_{Q} \rho(x) f(x, u) q d x=0, \quad \forall q \in W_{0}^{1, p(x)}(Q),
$$

for any domain $Q \Subset \Omega$, and $\max (k-u, 0) \in W_{0}^{1, p(x)}(\Omega)$ for every positive integer $k$.

Let $W_{0, \text { loc }}^{1, p(x)}(\Omega)=\left\{u \mid\right.$ there is an open domain $Q \Subset \Omega$ such that $\left.u \in W_{0}^{1, p(x)}(Q)\right\}$, and define $A: W_{\text {loc }}^{1, p(x)}(\Omega) \cap C(\Omega) \rightarrow\left(W_{0, l o c}^{1, p(x)}(\Omega)\right)^{*}$ as

$\langle A u, \varphi\rangle=\int_{\Omega}\left(|\nabla u|^{p(x)-2} \nabla u \nabla \varphi+\rho(x) e^{f(x, u)} \varphi\right) d x, \quad \forall u \in W_{\mathrm{loc}}^{1, p(x)}(\Omega) \cap C(\Omega), \forall \varphi \in W_{0, \mathrm{loc}}^{1, p(x)}(\Omega)$.

Lemma 2.1 (see $\left[9\right.$, Theorem 3.1]). Let $h \in W^{1, p(x)}(\Omega) \cap C(\Omega)$, and $X=h+W_{0}^{1, p(x)}(\Omega) \cap C(\Omega)$. Then, $A: X \rightarrow\left(W_{0, \text { loc }}^{1, p(x)}(\Omega)\right)^{*}$ is strictly monotone.

Letting $g \in\left(W_{0, \text { loc }}^{1, p(x)}(\Omega)\right)^{*}$, if $\langle g, \varphi\rangle \geq 0$,for all $\varphi \in W_{0, \text { loc }}^{1, p(x)}(\Omega)$ with $\varphi \geq 0$ a.e. in $\Omega$, then denote $g \geq 0$ in $\left(W_{0, \text { loc }}^{1, p(x)}(\Omega)\right)^{*}$; correspondingly, if $-g \geq 0$ in $\left(W_{0, \text { loc }}^{1, p(x)}(\Omega)\right)^{*}$, then denote $g \leq 0$ in $\left(W_{0, \text { loc }}^{1, p(x)}(\Omega)\right)^{*}$.

Definition 2.2. Let $u \in W_{\text {loc }}^{1, p(x)}(\Omega) \cap C(\Omega)$. If $A u \geq 0(A u \leq 0)$ in $\left(W_{0, \text { loc }}^{1, p(x)}(\Omega)\right)^{*}$, then $u$ is called a weak supersolution (weak subsolution) of $(\mathrm{P})$.

Copying the proof of [14], we have the following.

Lemma 2.3 (comparison principle). Let $u, v \in W_{\text {loc }}^{1, p(x)}(\Omega) \cap C(\Omega)$ satisfy

$$
A u-A v \geq 0, \quad \operatorname{in}\left(W_{0}^{1, p(x)}(\Omega)\right)^{*}
$$

Let $\varphi(x)=\min \{u(x)-v(x), 0\}$. If $\varphi(x) \in W_{0}^{1, p(x)}(\Omega)$ (i.e., $u \geq v$ on $\partial \Omega$ ), then $u \geq v$ a.e. in $\Omega$.

Lemma 2.4 (see [8, Theorem 1.1]). Under the conditions $\left(H_{1}\right)$ and $\left(H_{3}\right)$, if $u \in W^{1, p(x)}(\Omega)$ is a bounded weak solution of $-\Delta_{p(x)} u+\rho(x) e^{f(x, u)}=0$ in $\Omega$, then $u \in C_{\text {loc }}^{1, \vartheta}(\Omega)$, where $\vartheta \in(0,1)$ is a constant. 


\section{Asymptotic Behavior of Boundary Blow-Up Solutions}

If $u$ is a radial solution for $(\mathrm{P})$, then $(\mathrm{P})$ can be transformed into

$$
\begin{aligned}
& \left(r^{N-1}\left|u^{\prime}\right|^{p(r)-2} u^{\prime}\right)^{\prime}=r^{N-1} \rho(r) e^{f(r, u)}, \quad r \in(0, R), \\
& u(0)=u_{0}, \quad u^{\prime}(0)=0, \quad u^{\prime}(r) \geq 0, \quad \text { for } 0<r<R .
\end{aligned}
$$

It means that $u(r)$ is increasing.

Theorem 3.1. If $f(r, u)$ satisfies

$$
f(r, u) \geq \alpha u^{s} \quad(\text { as } u \longrightarrow+\infty) \text { for } r \in[\sigma, R) \text { uniformly }
$$

where $\sigma$ is defined in $\left(H_{4}\right)$ and $\alpha$ and s are positive constants, then there exists a supersolution $\Phi_{1}(x)$ which satisfies $\Phi_{1}(x) \rightarrow+\infty$ (as $d(x, \partial \Omega) \rightarrow 0$ ), such that for every solution $u$ of problem $(\mathrm{P})$, one has $u(x) \leq \Phi_{1}(x)$.

Proof. Define the function $g(r, a, \lambda)$ on $\left[0, R_{\curlywedge}\right)$ as

$$
g(r, a, \lambda)=\left\{\begin{array}{l}
\left(a \ln \frac{1}{(R-r)^{1-\theta}-\lambda}\right)^{1 / s}+k, \quad R_{0} \leq r<R_{\lambda} \\
k-\int_{r}^{R_{0}}\left[\frac{a^{1 / s}(1-\theta)\left(R-R_{0}\right)^{-\theta}}{s\left(\left(R-R_{0}\right)^{1-\theta}-\lambda\right)}\left(\ln \frac{1}{\left(R-R_{0}\right)^{1-\theta}-\lambda}\right)^{(1 / s)-1}\right]^{\left(p\left(R_{0}\right)-1\right) /(p(t)-1)} \\
\quad \times\left[\frac{\left(R_{0}\right)^{N-1}}{t^{N-1}} \sin \varepsilon(t-\sigma)\right]^{1 /(p(t)-1)} d t \\
\quad\left(a \ln \frac{1}{\left(R-R_{0}\right)^{1-\theta}-\lambda}\right)^{1 / s}, \sigma<r<R_{0}, \\
k-\int_{\sigma}^{R_{0}}\left[\frac{a^{1 / s}(1-\theta)\left(R-R_{0}\right)^{-\theta}}{s\left(\left(R-R_{0}\right)^{1-\theta}-\lambda\right)}\left(\ln \frac{1}{\left(R-R_{0}\right)^{1-\theta}-\lambda}\right)^{(1 / s)-1}\right]^{\left(p\left(R_{0}\right)-1\right) /(p(t)-1)} \\
\quad \times\left[\frac{\left(R_{0}\right)^{N-1}}{t^{N-1} \sin \varepsilon(t-\sigma)}\right]^{1 /(p(t)-1)} d t \\
+\left(a \ln \frac{1}{\left(R-R_{0}\right)^{1-\theta}-\lambda}\right)^{1 / s}, \quad r \leq \sigma,
\end{array}\right.
$$


where $\theta<\beta(R) / p(R), a>(1 / \alpha) \sup _{|x| \geq R_{0}} p(x)$ are constants, $R_{0} \in(\sigma, R), R-R_{0}$ is small enough, parameter $\lambda \in\left[0,\left(R-R_{0}\right)^{1-\theta} / 2\right], R_{\curlywedge}$ satisfies $\left(R-R_{\lambda}\right)^{1-\theta}-\lambda=0, \varepsilon=\pi / 2\left(R_{0}-\sigma\right)$

$$
\begin{aligned}
k=\left[\frac{2 p^{+}((1+s) / s+1 /(1-\theta))+|\beta|^{+} /(1-\theta)}{\alpha} \ln \frac{2}{\left(R-R_{0}\right)^{(1-\theta)}}\right]^{1 / s} \\
+\int_{\sigma}^{R_{0}}\left[\frac{2 a^{1 / s}(1-\theta)}{s\left(R-R_{0}\right)}\left(\ln \frac{2}{\left(R-R_{0}\right)^{1-\theta}}\right)^{(1 / s)-1}\right]^{\left(p\left(R_{0}\right)-1\right) /(p(t)-1)} \\
\times\left[\frac{\left(R_{0}\right)^{N-1}}{t^{N-1}} \sin \varepsilon(t-\sigma)\right]^{1 /(p(t)-1)} d t .
\end{aligned}
$$

Obviously, for any positive constant $a$, we have $g(r, a, \lambda) \in C^{1}\left[0, R_{\curlywedge}\right)$.

When $R_{0}<r<R_{\curlywedge}<R$, we have

$$
\begin{aligned}
& g^{\prime}=g^{\prime}(r, a, \lambda)=\frac{a^{1 / s}}{s}\left(\ln \frac{1}{(R-r)^{1-\theta}-\lambda}\right)^{(1 / s)-1} \frac{(1-\theta)(R-r)^{-\theta}}{(R-r)^{1-\theta}-\lambda}, \\
&\left|g^{\prime}\right|^{p(r)-2} g^{\prime}=\left[\frac{(1-\theta) a^{1 / s}}{s}\right]^{p(r)-1}\left(\ln \frac{1}{(R-r)^{1-\theta}-\lambda}\right)^{((1 / s)-1)(p(r)-1)} \frac{(R-r)^{-\theta(p(r)-1)}}{\left[(R-r)^{1-\theta}-\lambda\right]^{p(r)-1}}, \\
&\left(r^{N-1}\left|g^{\prime}\right|^{p(r)-2} g^{\prime}\right)^{\prime}= \\
& r^{N-1}\left[\frac{(1-\theta) a^{1 / s}}{s}\right]^{p(r)-1}\left(\ln \frac{1}{(R-r)^{1-\theta}-\lambda}\right)^{((1 / s)-1)(p(r)-1)} \\
& \times \frac{(p(r)-1)(R-r)^{-\theta p(r)}}{\left[(R-r)^{1-\theta}-\lambda\right]^{p(r)}}[(1-\theta)+\Pi(r)],
\end{aligned}
$$

where

$$
\begin{aligned}
\Pi(r)= & \frac{\left\{r^{N-1}\left[(1-\theta) a^{1 / s} / s\right]^{p(r)-1}\right\}^{\prime}}{(p(r)-1) r^{N-1}\left[(1-\theta) a^{1 / s} / s\right]^{p(r)-1}} \frac{(R-r)^{1-\theta}-\lambda}{(R-r)^{1-\theta}}(R-r)+\frac{((1 / s)-1)(1-\theta)}{\left(\ln \left(1 /\left((R-r)^{1-\theta}-\lambda\right)\right)\right)} \\
& +\frac{(R-r)^{1-\theta}-\lambda}{(R-r)^{1-\theta}}(R-r) \frac{((1 / s)-1) p^{\prime}(r)}{(p(r)-1)} \ln \left[\ln \frac{1}{(R-r)^{1-\theta}-\lambda}\right] \\
& +\frac{\theta p^{\prime}(r)}{(p(r)-1)} \frac{(R-r)^{1-\theta}-\lambda}{(R-r)^{1-\theta}}(R-r) \ln \frac{1}{(R-r)} \\
& +\theta \frac{(R-r)^{1-\theta}-\lambda}{(R-r)^{1-\theta}}+\frac{-p^{\prime}(r)}{p(r)-1}(R-r) \frac{(R-r)^{1-\theta}-\lambda}{(R-r)^{1-\theta}} \ln \left[(R-r)^{1-\theta}-\lambda\right] .
\end{aligned}
$$


If $\left(R-R_{0}\right)$ is small enough, it is easy to see that

$$
|\Pi(r)| \leq \ln \frac{1}{(R-r)^{1-\theta}-\lambda}, \quad \text { for } \mathcal{\lambda} \in\left[0, \frac{\left(R-R_{0}\right)^{1-\theta}}{2}\right] \text { uniformly, }
$$

and then

$$
\begin{aligned}
\left(r^{N-1}\left|g^{\prime}\right|^{p(r)-2} g^{\prime}\right)^{\prime} & \leq r^{N-1}\left[\frac{(1-\theta) a^{1 / s}}{s}\right]^{p(r)-1}\left(\ln \frac{1}{(R-r)^{1-\theta}-\lambda}\right)^{((1 / s)-1)(p(r)-1)+1} \\
& \times \frac{(p(r)-1)(R-r)^{-\theta p(r)}}{\left[(R-r)^{1-\theta}-\lambda\right]^{p(r)}}, \quad \forall r \in\left(R_{0}, R_{\curlywedge}\right) .
\end{aligned}
$$

Thus, when $0<R-R_{0}$ is small enough, from (3.5) and (3.8), for $\lambda \in\left[0,\left(R-R_{0}\right)^{1-\theta} / 2\right]$ uniformly, we have

$$
\begin{aligned}
& \left(r^{N-1}\left|g^{\prime}\right|^{p(r)-2} g^{\prime}\right)^{\prime} \\
& \quad \leq 2 r^{N-1}\left[\frac{(1-\theta) a^{1 / s}}{s}\right]^{p(r)-1}\left(\ln \frac{1}{(R-r)^{1-\theta}-\lambda}\right)^{((1 / s)-1)(p(r)-1)+1} \frac{(p(r)-1)(R-r)^{-\theta p(r)}}{\left[(R-r)^{1-\theta}-\lambda\right]^{p(r)}} \\
& \quad \leq r^{N-1} \rho(r)\left(\frac{1}{(R-r)^{1-\theta}-\lambda}\right)^{\alpha a}=r^{N-1} \rho(r) e^{\alpha g^{s}} \leq r^{N-1} \rho(r) e^{f(r, g)}, \quad \forall r \in\left(R_{0}, R_{\curlywedge}\right) .
\end{aligned}
$$

Thus, when $0<R-R_{0}$ is small enough, the following inequality is valid for $\lambda \in$ $\left[0,\left(R-R_{0}\right)^{1-\theta} / 2\right]$ uniformly:

$$
\left(r^{N-1}\left|g^{\prime}\right|^{p(r)-2} g^{\prime}\right)^{\prime} \leq r^{N-1} \rho(r) f(r, g), \quad \forall r \in\left(R_{0}, R_{\curlywedge}\right) .
$$


Obviously, if $R-R_{0}$ is small enough, then $g \geq\left[\left(\left(2 p^{+}((s+1) / s+1 /(1-\theta))+|\beta|^{+} /(1-\right.\right.\right.$ $\left.\theta)) / \alpha) \ln \left(2 /\left(R-R_{0}\right)^{1-\theta}\right)\right]^{1 / s}$ is large enough. Since $\lambda \in\left[0,\left(R-R_{0}\right)^{1-\theta} / 2\right]$,

$$
\begin{aligned}
& \left(r^{N-1}\left|g^{\prime}\right|^{p(r)-2} g^{\prime}\right)^{\prime} \\
& \quad=\varepsilon\left(R_{0}\right)^{N-1}\left[\frac{a^{1 / s}(1-\theta)\left(R-R_{0}\right)^{-\theta}}{s\left(\left(R-R_{0}\right)^{1-\theta}-\lambda\right)}\left(\ln \frac{1}{\left(R-R_{0}\right)^{1-\theta}-\lambda}\right)^{(1 / s)-1}\right]^{\left(p\left(R_{0}\right)-1\right)} \cos (\varepsilon(r-\sigma)) \\
& \quad \leq \varepsilon\left(R_{0}\right)^{N-1}\left[\frac{a^{1 / s}(1-\theta)\left(R-R_{0}\right)^{-\theta}}{s(1 / 2)\left(R-R_{0}\right)^{1-\theta}}\left(\ln \frac{2}{\left(\mathrm{R}-R_{0}\right)^{1-\theta}}\right)^{(1 / s)+1}\right]^{\left(p\left(R_{0}\right)-1\right)} \\
& \quad \leq \varepsilon\left(R_{0}\right)^{N-1}\left[\frac{2 a^{1 / s}(1-\theta)}{s\left(R-R_{0}\right)}\left(\frac{2}{\left(R-R_{0}\right)^{1-\theta}}\right)^{(1 / s)+1}\right]^{\left(p\left(R_{0}\right)-1\right)} \\
& \quad \leq \varepsilon\left(R_{0}\right)^{N-1}\left[\frac{2 a^{1 / s}(1-\theta)}{s}\left(\frac{2}{R-R_{0}}\right)^{((s+1) / s)(1-\theta)+1}\right]^{p^{+}} \\
& \quad \leq r^{N-1} \rho(r) e^{\alpha g^{s}} \leq r^{N-1} \rho(r) e^{f(r, g)}, \quad \sigma<r<R_{0} .
\end{aligned}
$$

Thus,

$$
\left(r^{N-1}\left|g^{\prime}\right|^{p(r)-2} g^{\prime}\right)^{\prime} \leq r^{N-1} \rho(r) e^{f(r, g)}, \quad \sigma<r<R_{0} .
$$

Obviously,

$$
\left(r^{N-1}\left|g^{\prime}\right|^{p(r)-2} g^{\prime}\right)^{\prime}=0 \leq r^{N-1} \rho(r) e^{f(r, g)}, \quad 0 \leq r<\sigma .
$$

Since $g(x, a, \lambda)=g(|x|, a, \lambda)$ is a $C^{1}$ function on $B\left(0, R_{\curlywedge}\right)$, if $0<R-R_{0}$ is small enough $\left(R_{0}\right.$ depends on $\left.R, p, s, \alpha\right)$, from (3.10), (3.12), and (3.13), for any $\lambda \in\left[0,\left(R-R_{0}\right)^{1-\theta} / 2\right]$, we can see that $g(|x|, a, \lambda)$ is a supersolution for $(\mathrm{P})$ on $B\left(0, R_{\curlywedge}\right)$, and then $g(|x|, a, 0)$ is a supersolution for $(\mathrm{P})$.

Defining the function $g_{m}(|x|, a-\epsilon)=g(r, a-\epsilon, 1 / m)$ on $\left[0, R_{1 / m}\right)$, where $a-\epsilon>$ $(1 / \alpha) \sup _{|x| \geq R_{0}} p(x)$, then $g_{m}(|x|, a-\epsilon)$ is a supersolution for $(\mathrm{P})$ on $B(0, R-(1 / m))$. If $u$ is a solution for $(\mathrm{P})$, according to the comparison principle, we get that $g_{m}(|x|, a-\epsilon) \geq u(x)$ for any $x \in B\left(0, R_{1 / m}\right)$. For any $x \in B(0, R) \backslash B\left(0, R_{0}\right)$, we have $g_{m}(|x|, a-\epsilon) \geq g_{m+1}(|x|, a-\epsilon)$, when $m$ is large enough. Thus

$$
u(x) \leq \lim _{m \longrightarrow+\infty} g_{m}(|x|, a-\epsilon), \quad \forall x \in B(0, R) \backslash B\left(0, R_{0}\right)
$$


When $d(x, \partial \Omega)>0$ is small enough, we have

$$
\lim _{m \rightarrow+\infty} g_{m}(|x|, a-\epsilon)<\left(a \ln \frac{1}{(R-r)^{1-\theta}}\right)^{1 / s}+k \leq g(|x|, a, 0) .
$$

According to the comparison principle, we get that $g(|x|, a, 0) \geq u(x)$, for all $x \in$ $B(0, R)$; then $\Phi_{1}(x)=\Phi_{1}(|x|)=g(|x|, a, 0)$ is a radial upper control function of all of the solutions for $(\mathrm{P})$, and $\Phi_{1}(x)=\Phi_{1}(|x|)$ is a radial supersolution for $(\mathrm{P})$. The proof is completed.

Theorem 3.2. If $f(r, u)$ satisfies

$$
\begin{gathered}
f(r, u) \longrightarrow-\infty \quad(\text { as } u \longrightarrow-\infty) \text { for } r \in[\sigma, R) \text { uniformly, } \\
f(r, u) \leq \delta u^{s} \quad(\text { as } u \longrightarrow+\infty) \text { for } r \in[\sigma, R) \text { uniformly, }
\end{gathered}
$$

where $\sigma$ is defined in $\left(H_{4}\right)$ and $\delta$ and $s$ are positive constants, then there exists a subsolution $\Phi_{2}(x)$ which satisfies $\Phi_{2}(x) \rightarrow+\infty($ as $d(x, \partial \Omega) \rightarrow 0)$, such that for every solution $u(x)$ for problem $(\mathrm{P})$, one has $u(x) \geq \Phi_{2}(x)$.

Proof. We will prove this theorem in the following two cases.

(i) $\beta_{1}(R)>0$.

(ii) $\beta_{1}(R) \leq 0$.

Case $1\left(\beta_{1}(R)>0\right)$. Let $z_{1}$ be a radial solution of

$$
-\Delta_{p(x)} z_{1}(x)=-\mu, \quad \text { in } \Omega_{1}=B(0, \sigma), \quad z_{1}=0, \quad \text { on } \partial \Omega_{1},
$$

where $\mu>2\left(\max _{r \in\left[0, R_{0}\right]} \rho(r)+1\right)^{2\left(p^{+}-1\right) /\left(p^{-}-1\right)}$ is a positive constant. We denote $z_{1}=z_{1}(r)=$ $z_{1}(|x|)$. Then, $z_{1}$ satisfies

$$
\begin{array}{r}
-\left(r^{N-1}\left|z_{1}^{\prime}\right|^{p(r)-2} z_{1}^{\prime}\right)^{\prime}=-r^{N-1} \mu, \quad z_{1}(\sigma)=0, \quad z_{1}^{\prime}(0)=0, \\
z_{1}^{\prime}=\left|\frac{r \mu}{N}\right|^{1 /(p(r)-1)}, z_{1}=-\int_{r}^{\sigma}\left|\frac{r \mu}{N}\right|^{1 /(p(r)-1)} d r .
\end{array}
$$

Denote $h_{b}(r, \lambda)$ on $\left[\sigma, R_{0}\right]$ as

$$
\begin{aligned}
h_{b}(r, \lambda)=\int_{r}^{R_{0}}\{ & \frac{\left(R_{0}\right)^{N-1}}{t^{N-1}} \frac{t-\sigma}{R_{0}-\sigma}\left[\frac{b(1-\theta)\left(R-R_{0}\right)^{-\theta}}{s\left(\left(R-R_{0}\right)^{1-\theta}+\lambda\right)}\left(b \ln \frac{1}{\left(R-R_{0}\right)^{1-\theta}+\lambda}\right)^{(1 / s)-1}\right]^{p\left(R_{0}\right)-1} \\
& \left.+\frac{(\sigma)^{N-1}}{t^{N-1}} \frac{R_{0}-t}{R_{0}-\sigma}\left|\frac{\sigma \mu}{N}\right|\right\}^{1 /(p(t)-1)} d t .
\end{aligned}
$$


It is easy to see that

$$
\begin{gathered}
-h_{b}^{\prime}(\sigma, \lambda)=z_{1}^{\prime}(\sigma)=\left|\frac{\sigma \mu}{N}\right|^{1 /(p(\sigma)-1)}, \\
-h_{b}^{\prime}\left(R_{0}, \lambda\right)=\frac{b(1-\theta)\left(R-R_{0}\right)^{-\theta}}{s\left(\left(R-R_{0}\right)^{1-\theta}+\lambda\right)}\left(b \ln \frac{1}{\left(R-R_{0}\right)^{1-\theta}+\lambda}\right)^{(1 / s)-1} .
\end{gathered}
$$

Define the function $v(r, b, \lambda)$ on $[0, R)$ as

$$
v(r, b, \lambda)= \begin{cases}\left(b \ln \frac{1}{(R-r)^{1-\theta}+\lambda}\right)^{1 / s}-k^{*}, & R_{0} \leq r<R, \\ \left(b \ln \frac{1}{\left(R-R_{0}\right)^{1-\theta}+\lambda}\right)^{1 / s}-k^{*}-h_{b}(r, \lambda), & \sigma<r<R_{0}, \\ -\int_{r}^{\sigma}\left|\frac{r \mu}{N}\right|^{1 /(p(r)-1)} d r+\left(b \ln \frac{1}{\left(R-R_{0}\right)^{1-\theta}+\lambda}\right)^{1 / s}-k^{*}-h_{b}(\sigma, \lambda), & r \leq \sigma,\end{cases}
$$

where $\theta \in\left(\beta_{1}(R) / p(R), 1\right), b \in\left(0,(1 / \delta) \inf _{|x| \geq R_{0}} p(x)\right)$ are constants, $R_{0} \in(\sigma, R), R-R_{0}$ is small enough, parameter $\lambda \in\left[0,\left(R-R_{0}\right)^{1-\theta} / 2\right]$, and

$$
k^{*}=M+\left(b \ln \frac{1}{\left(R-R_{0}\right)^{1-\theta}}\right)^{1 / s}
$$

where $M$ satisfies

$$
(\sigma)^{N-1} \frac{1}{R_{0}-\sigma} \geq r^{N-1} \rho(r) e^{f(r, y)}, \quad \forall y \leq-M, \forall r \in\left[0, R_{0}\right]
$$

Obviously, for any positive constant $b, v(r, b, \lambda) \in C^{1}[0, R)$.

By computation, when $r \in\left(R_{0}, R\right)$, we have

$$
\begin{gathered}
v^{\prime}=v^{\prime}(r, b, \lambda)=\frac{b^{1 / s}}{s}\left(\ln \frac{1}{(R-r)^{1-\theta}+\lambda}\right)^{1 / s-1} \frac{(1-\theta)(R-r)^{-\theta}}{(R-r)^{1-\theta}+\lambda}, \\
\left|v^{\prime}\right|^{p(r)-2} v^{\prime}=\left[\frac{(1-\theta) b^{1 / s}}{s}\right]^{p(r)-1}\left(\ln \frac{1}{(R-r)^{1-\theta}+\lambda}\right)^{(1 / s-1)(p(r)-1)} \frac{(R-r)^{-\theta(p(r)-1)}}{\left[(R-r)^{1-\theta}+\lambda\right]^{p(r)-1}},
\end{gathered}
$$


Abstract and Applied Analysis

$$
\begin{aligned}
\left(r^{N-1}\left|v^{\prime}\right|^{p(r)-2} v^{\prime}\right)^{\prime}= & r^{N-1}\left[\frac{(1-\theta) b^{1 / s}}{s}\right]^{p(r)-1}\left(\ln \frac{1}{(R-r)^{1-\theta}+\lambda}\right)^{(1 / s-1)(p(r)-1)} \\
& \times \frac{(p(r)-1)(R-r)^{-\theta(p(r)-1)-1}}{\left[(R-r)^{1-\theta}+\lambda\right]^{p(r)-1}}(\theta+\Lambda(r))
\end{aligned}
$$

where

$$
\begin{aligned}
\Lambda(r)= & \frac{\left\{r^{N-1}\left[(1-\theta) b^{1 / s} / s\right]^{p(r)-1}\right\}^{\prime}}{(p(r)-1) r^{N-1}\left[(1-\theta) b^{1 / s} / s\right]^{p(r)-1}}(R-r)+\frac{(1 / s-1)(1-\theta)}{\left(\ln \left(1 /\left((R-r)^{1-\theta}+\lambda\right)\right)\right)\left[(R-r)^{1-\theta}+\lambda\right]} \\
& \times(R-r)^{1-\theta}+\frac{(1 / s-1) p^{\prime}(r)}{(p(r)-1)}(R-r) \ln \left[\ln \frac{1}{(R-r)^{1-\theta}+\lambda}\right]+\frac{\theta p^{\prime}(r)}{(p(r)-1)}(R-r) \ln \frac{1}{(R-r)} \\
& +\frac{(1-\theta)}{\left[(R-r)^{1-\theta}+\lambda\right]}(R-r)^{1-\theta}+\frac{-p^{\prime}(r)}{p(r)-1}(R-r) \ln \left[(R-r)^{1-\theta}+\lambda\right] .
\end{aligned}
$$

By computation, when $R-R_{0}$ is small enough, for $\lambda \in\left[0,\left(R-R_{0}\right)^{1-\theta} / 2\right]$ uniformly, we have

$$
\begin{aligned}
\left(r^{N-1}\left|v^{\prime}\right|^{p(r)-2} v^{\prime}\right)^{\prime} & \\
\geq & r^{N-1}\left[\frac{(1-\theta) b^{1 / s}}{s}\right]^{p(r)-1}\left(\ln \frac{1}{(R-r)^{1-\theta}+\lambda}\right)^{(1 / s-1)(p(r)-1)} \\
& \times \frac{(p(r)-1)(R-r)^{-\theta(p(r)-1)-1}}{\left[(R-r)^{1-\theta}+\lambda\right]^{p(r)-1}} \theta\left(1-\frac{1}{2}\right) \\
\geq & \frac{\theta}{2} r^{N-1}\left[\frac{(1-\theta) b^{1 / s}}{s}\right]^{p(r)-1}\left(\ln \frac{1}{(R-r)^{1-\theta}+\lambda}\right)^{(1 / s-1)(p(r)-1)} \\
& \left.\times \frac{(p(r)-1)(R-r)^{-\theta(p(r)-1)-1}(R-r)^{1-\theta}}{\left[(R-r)^{1-\theta}+\lambda\right]^{p(r)}(R)}\right)^{(1 / s-1)(p(r)-1)} \frac{(p(r)-1)(R-r)^{-\theta p(r)}}{\left[(R-r)^{1-\theta}+\lambda\right]^{p(r)}} \\
\geq & \frac{\theta}{2} r^{N-1}\left[\frac{(1-\theta) b^{1 / s}}{s}\right]^{p(r)-1}\left(\ln \frac{1}{(R-r)^{1-\theta}+\lambda}\right)^{\geq} \\
\geq & r^{N-1} \rho_{1}(R-r)^{-\beta_{1}(r)} e^{\delta(r) e^{f(r, v)}, \quad \forall r \in\left(R_{0}, R\right) .}
\end{aligned}
$$


Then, for $\lambda \in\left[0,\left(R-R_{0}\right)^{1-\theta} / 2\right]$ uniformly, we have

$$
\left(r^{N-1}\left|v^{\prime}\right|^{p(r)-2} v^{\prime}\right)^{\prime} \geq r^{N-1} \rho(r) e^{f(r, v)}, \quad \forall r \in\left(R_{0}, R\right)
$$

When $R-R_{0}$ is small enough, for all $r \in\left(\sigma, R_{0}\right)$, since $v \leq-M$, it is easy to see that

$$
\begin{aligned}
\left(r^{N-1}\left|v^{\prime}\right|^{p(r)-2} v^{\prime}\right)^{\prime} \geq & \left(r^{N-1}\left|h^{\prime}\right|^{p(r)-2} h^{\prime}\right)^{\prime} \\
= & \left(R_{0}\right)^{N-1} \frac{1}{R_{0}-\sigma}\left[\frac{b(1-\theta)\left(R-R_{0}\right)^{-\theta}}{s\left(\left(R-R_{0}\right)^{1-\theta}+\lambda\right)}\left(b \ln \frac{1}{\left(R-R_{0}\right)^{1-\theta}+\lambda}\right)^{1 / s-1}\right]^{p\left(R_{0}\right)-1} \\
& -(\sigma)^{N-1} \frac{1}{R_{0}-\sigma}\left|\frac{\sigma \mu}{N}\right| \\
\geq & (\sigma)^{N-1} \frac{1}{R_{0}-\sigma} \\
\geq & r^{N-1} \rho(r) e^{f(r, v)},
\end{aligned}
$$

Then,

$$
\left(r^{N-1}\left|v^{\prime}\right|^{p(r)-2} v^{\prime}\right)^{\prime} \geq r^{N-1} \rho(r) e^{f(r, v)}, \quad \forall r \in\left(\sigma, R_{0}\right)
$$

Obviously,

$$
\left(r^{N-1}\left|v^{\prime}\right|^{p(r)-2} v^{\prime}\right)^{\prime}=r^{N-1} \mu \geq r^{N-1} \rho(r) e^{f(r, v)}, \quad \forall r \in(0, \sigma)
$$

Combining (3.27), (3.29), and (3.30), when $R-R_{0}$ is large enough, for any $\lambda \in[0,(R-$ $\left.R_{0}\right)^{1-\theta} / 2$ ], one can see that $v(r, a, \lambda)$ is a subsolution for $(\mathrm{P})$.

Define the function $v_{m}(r, b+\epsilon)$ on $B(0, R)$ as

$$
v_{m}(r, b+\epsilon)=v_{m}\left(r, b+\epsilon, \frac{1}{m}\right)
$$

where $\epsilon$ is a small enough positive constant such that $(b+\epsilon)<(1 / \delta) \inf _{|x| \geq R_{0}} p(x)$.

For any $m=1,2, \ldots$, we can see that $v_{m}(r, b+\epsilon) \in C^{1}([0, R))$ is a subsolution for (P) on $B\left(R_{0}, R\right)$. According to the comparison principle, we get that $v_{m}(r, b+\epsilon) \leq u(x)$ for any $x \in B(0, R)$. For any $x \in B(0, R) \backslash B\left(0, R_{0}\right)$, we have $v_{m}(|x|, b+\epsilon) \leq v_{m+1}(|x|, b+\epsilon)$. Thus

$$
u(x) \geq \lim _{m \longrightarrow+\infty} v_{m}(|x|, b+\epsilon), \quad \forall x \in B(0, R) \backslash B\left(0, R_{0}\right) .
$$

When $d(x, \partial \Omega)$ is small enough, we have $\lim _{m \longrightarrow+\infty} v_{m}(|x|, b+\epsilon)>v(|x|, b, 0)$. 
According to the comparison principle, we get that $v(|x|, b, 0) \leq u(x), \forall x \in B(0, R)$; then $\Phi_{2}(x)=\Phi_{2}(|x|)=v(|x|, b, 0)$ is a radial lower control function of all of the solutions for $(\mathrm{P})$, and $\Phi_{2}(x)$ is a radial subsolution for $(\mathrm{P})$.

Case $2\left(\beta_{1}(R) \leq 0\right)$. Let $\mu>2\left(\max _{r \in\left[0, R_{0}\right]} \rho(r)+1\right)^{2\left(p^{+}-1\right) /\left(p^{-}-1\right)}$ be a positive constant. Denote $\varpi_{b}(r, \lambda)$ on $\left[\sigma, R_{0}\right]$ as

$$
\begin{aligned}
\varpi_{b}(r, \lambda)=\int_{r}^{R_{0}}\{ & \frac{\left(R_{0}\right)^{N-1}}{t^{N-1}} \frac{t-\sigma}{R_{0}-\sigma}\left[\frac{b}{s\left(R+\lambda-R_{0}\right)}\left(b \ln \left(R+\lambda-R_{0}\right)^{-1}\right)^{1 / s-1}\right]^{p\left(R_{0}\right)-1} \\
& \left.+\frac{(\sigma)^{N-1}}{t^{N-1}} \frac{R_{0}-t}{R_{0}-\sigma}\left|\frac{\sigma \mu}{N}\right|\right\}^{1 /(p(t)-1)} d t .
\end{aligned}
$$

It is easy to see that

$$
-\varpi_{b}^{\prime}(\sigma, \lambda)=z_{1}^{\prime}(\sigma)=\left|\frac{\sigma \mu}{N}\right|^{1 /(p(\sigma)-1)}, \quad-\varpi_{b}^{\prime}\left(R_{0}, \lambda\right)=\frac{b}{s\left(R+\lambda-R_{0}\right)}\left(b \ln \left(R+\lambda-R_{0}\right)^{-1}\right)^{1 / s-1} .
$$

Define the function $\eta(r, b, \lambda)$ on $B(0, R)$ as

$$
\eta(r, b, \lambda)= \begin{cases}\left(b \ln (R+\lambda-r)^{-1}\right)^{1 / s}-k^{*}, & R_{0} \leq r<R, \\ \left(b \ln \left(R+\lambda-R_{0}\right)^{-1}\right)^{1 / s}-k^{*}-\varpi_{b}(r, \lambda), & \sigma<r<R_{0}, \\ -\int_{r}^{\sigma}\left|\frac{r \mu}{N}\right|^{1 /(p(r)-1)} d r+\left(b \ln \left(R+\lambda-R_{0}\right)^{-1}\right)^{1 / s}-k^{*}-\varpi_{b}(\sigma, \lambda), & r \leq \sigma,\end{cases}
$$

where $b \in\left(0,(1 / \delta) \inf _{|x| \geq R_{0}}\left[p(x)-\beta_{1}(x)\right]\right)$ is a constant, $R_{0} \in(\sigma, R), R-R_{0}$ is small enough, parameter $\lambda \in\left[0,\left(R-R_{0}\right) / 2\right]$, and

$$
k^{*}=M+\left(b \ln \frac{1}{R-R_{0}}\right)^{1 / s}
$$

where $M$ is defined in (3.23).

Obviously, for any positive constant $b, \eta(r, b, \lambda) \in C^{1}[0, R)$. 
Similar to the proof of Case 1 , when $R-R_{0}$ is small enough, we have

$$
\begin{aligned}
& \left(r^{N-1}\left|\eta^{\prime}\right|^{p(r)-2} \eta^{\prime}\right)^{\prime} \\
& \quad \geq r^{N-1}\left(\frac{b^{1 / s}}{s}\right)^{p(r)-1}(p(r)-1)(R+\lambda-r)^{-p(r)}\left(\ln (R+\lambda-r)^{-1}\right)^{(1 / s-1)(p(r)-1)}\left(1-\frac{1}{2}\right) \\
& \quad \geq r^{N-1} \rho(r) e^{f(r, \eta)}, \quad \forall r \in\left(R_{0}, R\right) .
\end{aligned}
$$

When $R-R_{0}$ is small enough, for all $r \in\left(\sigma, R_{0}\right)$, from the definition of $k^{*}$, it is easy to see that

$$
\left(r^{N-1}\left|\eta^{\prime}\right|^{p(r)-2} \eta^{\prime}\right)^{\prime} \geq(\sigma)^{N-1} \frac{1}{R_{0}-\sigma} \geq r^{N-1} \rho(r) e^{f(r, \eta)} .
$$

Obviously

$$
\left(r^{N-1}\left|\eta^{\prime}\right|^{p(r)-2} \eta^{\prime}\right)^{\prime}=r^{N-1} \mu \geq r^{N-1} \rho(r) e^{f(r, \eta)}, \quad \forall r \in(0, \sigma) .
$$

Combining (3.37), (3.38), and (3.39), when $R-R_{0}$ is large enough, for any $\lambda \in[0,(R-$ $\left.R_{0}\right) / 2$ ], one can see that $\eta(r, a, \lambda)$ is a subsolution for $(\mathrm{P})$.

Define the function $\eta_{m}(r, b+\varepsilon)$ on $B(0, R)$ as

$$
\eta_{m}(r, b+\varepsilon)=\eta\left(r, b+\varepsilon, \frac{1}{m}\right)
$$

where $\varepsilon$ is a small enough positive constant such that $(b+\varepsilon)<(1 / \delta) \inf _{|x| \geq R_{0}} p(x)$.

We can see that $\eta_{m}(r, b+\varepsilon) \in C^{1}[0, R)$ is a subsolution for $(\mathrm{P})$ for any $m=1,2 \ldots$ According to the comparison principle, we get that $\eta_{m}(r, b+\varepsilon) \leq u(x)$ for any $x \in B(0, R)$. For any $x \in B(0, R) \backslash B\left(0, R_{0}\right)$, we have $\eta_{m}(|x|, b+\varepsilon) \leq \eta_{m+1}(|x|, b+\varepsilon)$. Then,

$$
u(x) \geq \lim _{m \rightarrow+\infty} \eta_{m}(|x|, b+\varepsilon), \quad \forall x \in B(0, R) \backslash B\left(0, R_{0}\right) .
$$

When $d(x, \partial \Omega)$ is small enough, we have

$$
\lim _{m \longrightarrow+\infty} \eta_{m}(|x|, b+\varepsilon)>\eta(|x|, b, 0) .
$$

According to the comparison principle, we get that $\eta(|x|, b, 0) \leq u(x), \forall x \in B(0, R)$; then $\Phi_{2}(x)=\Phi_{2}(|x|)=\eta(|x|, b, 0)$ is a radial lower control function of all of the solutions for $(\mathrm{P})$, and $\Phi_{2}(x)=\Phi_{2}(|x|)$ is a radial subsolution for $(\mathrm{P})$. 
Theorem 3.3. If $f(r, u)$ satisfies

$$
\lim _{u \longrightarrow+\infty} \frac{f(r, u)}{u^{s}}=\delta \quad(\text { as } u \longrightarrow+\infty) \text { for } r \in[\sigma, R) \text { uniformly, }
$$

where $\sigma$ is defined in $\left(H_{4}\right), \delta$ and s are positive constants, $\rho(r)=\rho_{0}(R-r)^{-\beta(r)}$, where $\beta(R)<p(R)$, then each solution $u(x)$ for $(\mathrm{P})$ satisfies

$$
\lim _{|x| \rightarrow R} \frac{u(x)}{\left((p(R) / \delta)\left(\ln 1 /(R-|x|)^{1-\theta}\right)\right)^{1 / s}}=1, \quad \text { where } \theta=\frac{\beta(R)}{p(R)}
$$

Proof. It is easy to be seen from Theorems 3.1 and 3.2

\section{The Existence of Boundary Blow-Up Solutions}

Theorem 4.1. If $\inf _{x \in \Omega} p(x)>N$ and $f(r, u)$ satisfies

$$
f(r, u) \geq a u^{s} \quad(\text { as } u \rightarrow+\infty) \text { for } r \in[\sigma, R) \text { uniformly, }
$$

where $\sigma$ is defined in $\left(H_{4}\right), a$ and s are positive constants, then $(\mathrm{P})$ possesses a boundary blow-up solution.

Proof. In order to deal with the existence of boundary blow-up solutions, let us consider the problem

$$
\begin{gathered}
-\Delta_{p(x)} u+\rho(r) e^{f(x, u)}=0, \quad \text { in } \Omega_{0}, \\
u(x)=c, \quad \text { for } x \in \partial \Omega_{0}
\end{gathered}
$$

where $c$ is a positive constant and $\Omega_{0} \Subset \Omega$ is a radial subdomain of $\Omega$. Since $\inf _{x \in \Omega} p(x)>N$, then $W^{1, p(x)}\left(\Omega_{0}\right) \hookrightarrow C^{\alpha}\left(\overline{\Omega_{0}}\right)$, where $\alpha \in(0,1)$. The relative functional of $(4.2)$ is

$$
\varphi=\int_{\Omega_{0}} \frac{1}{p(x)}|\nabla u(x)|^{p(x)} d x+\int_{\Omega_{0}} F(x, u) d x,
$$

where $F(x, u)=\int_{0}^{u} e^{f(x, t)} d t$. Since $\varphi$ is coercive in $X:=c+W_{0}^{1, p(x)}\left(\Omega_{0}\right)$, then $\varphi$ possesses a nontrivial minimum point $u$. So, problem (4.2) possesses a weak solution $u$.

Since $a u^{s} \leq f(r, u) \leq C_{1}+C_{2}|u|^{r(x)}$, from Theorems 3.1 and 3.2, we get that $(\mathrm{P})$ possesses a supersolution $g^{*}(x)$ and a subsolution $g_{*}(x)$, which satisfy $g^{*}(x) \geq g_{*}(x)$, when $d(x, \partial \Omega)$ (the distance from $x$ to $\partial \Omega$ ) is small enough. According to the comparison principle, we get that $g^{*}(x) \geq g_{*}(x)$ for any $x \in \Omega$. 
Denote $D_{j}=\{x|| x \mid<1-1 /(j+1) R\}(j=1,2, \ldots)$. Let us consider the problem

$$
\begin{gathered}
-\Delta_{p(x)} u_{j}+\rho(x) e^{f\left(x, u_{j}\right)}=0, \quad \text { in } D_{j}, \\
u_{j}(x)=g_{*}(x), \quad \text { for } x \in \partial D_{j},
\end{gathered}
$$

and the relative functional is

$$
\varphi=\int_{D_{j}} \frac{1}{p(x)}\left|\nabla u_{j}(x)\right|^{p(x)} d x+\int_{D_{j}} \rho(x) F\left(x, u_{j}\right) d x .
$$

Let $g_{* j}(x)=\left.g_{*}(x)\right|_{D_{i}}$. Since the functional $\varphi$ is coercive in $X_{j}=g_{* j}(x)+W_{0}^{1, p(x)}\left(D_{j}\right)$, then $\varphi$ has a nontrivial minimum point $u_{j}$. Therefore, problem (4.4) has a weak solution $u_{j}$.

According to the comparison principle, we get that $g_{*}(x) \leq u_{j}(x)$ for any $x \in D_{j}$ $(j=1,2, \ldots)$. Since $u_{j}(x)=g_{*}(x)$ for any $x \in \partial D_{j}$, then $u_{j}(x) \leq u_{j+1}(x)$ for any $x \in \partial D_{j}$ $(j=1,2, \ldots)$. According to the comparison principle, we get that $u_{j}(x) \leq u_{j+1}(x)$ for any $x \in D_{j}(j=1,2, \ldots)$.

Since $g^{*}(x)$ is a supersolution and $g^{*}(x) \geq g_{*}(x)$ for any $x \in \Omega$, so we have $u_{j}(x)=$ $g_{*}(x) \leq g^{*}(x)$ for any $x \in \partial D_{j}(j=1,2, \ldots)$. According to the comparison principle, we get that $u_{j}(x) \leq g^{*}(x)$ for any $x \in D_{j}(j=1,2, \ldots)$.

Since $g^{*}(x)$ and $g_{*}(x)$ are locally bounded, from Lemma 2.4, each weak solution of (4.4) is a $C_{\text {loc }}^{1, \alpha}$ function. The $C^{1, \alpha}$ interior regularity result implies that the sequences $\left\{u_{j}\right\}$ and $\left\{\nabla u_{j}\right\}$ are equicontinuous in $D_{2}$, and hence we can choose a subsequence, which we denoted by $\left\{u_{j}^{1}\right\}$, such that $u_{j}^{1} \rightarrow w_{1}$ and $\nabla u_{j}^{1} \rightarrow \varpi_{1}$ uniformly on $D_{1}$ for some $w_{1} \in C\left(D_{1}\right)$ and $\varpi_{1} \in\left(C\left(D_{1}\right)\right)^{N}$. In fact, $\varpi_{1}=\nabla w_{1}$ on $D_{1}$, and from the interior $C^{1, \alpha}$ estimate, we conclude that $\nabla w_{1} \in\left(C^{\alpha}\left(D_{1}\right)\right)^{N}$ for some $0<\alpha<1$. Thus, $w_{1} \in W^{1, p(x)}\left(D_{1}\right) \cap C^{1, \alpha}\left(D_{1}\right)$. From the $C^{1, \alpha}$ interior regularity result, we see that $\left|\nabla u_{j}\right|^{p-1}|\nabla \varphi| \leq C|\nabla \varphi|$ on $D_{1}$, and since the function $\xi \rightarrow|\xi|^{p-2} \xi$ is continuous on $\mathbb{R}^{N}$, it follows that $\left|\nabla u_{j}^{1}(x)\right|^{p-2} \nabla u_{j}^{1}(x) \cdot \nabla \varphi(x) \rightarrow\left|\nabla w_{1}(x)\right|^{p-2} \nabla w_{1}(x) \cdot \nabla \varphi(x)$ for $x \in D_{1}$. Thus, by the dominated convergence theorem, we have

$$
\int_{D_{1}}\left|\nabla u_{j}^{1}(x)\right|^{p-2} \nabla u_{j}^{1}(x) \cdot \nabla \varphi(x) d x \longrightarrow \int_{D_{1}}\left|\nabla w_{1}(x)\right|^{p-2} \nabla w_{1}(x) \cdot \nabla \varphi(x) d x, \quad \forall \varphi \in W_{0}^{1, p(x)}\left(D_{1}\right) .
$$

Furthermore, since $0 \leq f\left(u_{j}^{1}\right) \leq f\left(u_{j+1}^{1}\right)$ and $f\left(u_{j}^{1}(x)\right) \rightarrow f\left(w_{1}(x)\right)$ for each $x \in D_{1}$, by the monotone convergence theorem, we obtain

$$
\int_{D_{1}} \rho e^{f\left(u_{j}^{1}\right)} q d x \longrightarrow \int_{D_{1}} \rho e^{f\left(w_{1}\right)} q d x, \quad \forall q \in W_{0}^{1, p(x)}\left(D_{1}\right)
$$

Therefore, it follows that

$$
\int_{D_{1}}\left|\nabla w_{1}(x)\right|^{p-2} \nabla w_{1}(x) \cdot \nabla q(x) d x+\int_{D_{1}} \rho e^{f\left(w_{1}\right)} q d x=0, \quad \forall q \in W_{0}^{1, p(x)}\left(D_{1}\right),
$$

and hence $w_{1}$ is a weak solution for $-\Delta_{p(x)} w_{1}+\rho e^{f\left(w_{1}\right)}=0$ on $D_{1}$. 
Thus, there exists a subsequence of $\left\{u_{j}\right\}$ which we denote it by $\left\{u_{j}^{1}\right\}$, such that $u_{j}^{1} \rightarrow w_{1}$ in $D_{1}($ as $j \rightarrow \infty)$, where $w_{1} \in W^{1, p(x)}\left(D_{1}\right) \cap C^{1, \alpha_{1}}\left(D_{1}\right)$ and satisfies

$$
\int_{D_{1}}\left|\nabla w_{1}\right|^{p(x)-2} \nabla w_{1} \nabla q d x+\int_{D_{1}} \rho(x) e^{f\left(x, w_{1}\right)} q d x=0, \quad \forall q \in W_{0}^{1, p(x)}\left(D_{1}\right)
$$

Similarly, we can prove that there exists a subsequence of $\left\{u_{j}^{1}\right\}$ which we denote by $\left\{u_{j}^{2}\right\}$, such that $u_{j}^{2} \rightarrow w_{2}$ in $D_{2}($ as $j \rightarrow \infty)$, where $w_{2} \in W^{1, p(x)}\left(D_{2}\right) \cap C^{1, \alpha_{2}}\left(D_{2}\right)$ satisfies $w_{1}=\left.w_{2}\right|_{D_{1}}$ and

$$
\int_{D_{2}}\left|\nabla w_{2}\right|^{p(x)-2} \nabla w_{2} \nabla q d x+\int_{D_{2}} \rho(x) e^{f\left(x, w_{2}\right)} q d x=0, \quad \forall q \in W_{0}^{1, p(x)}\left(D_{2}\right)
$$

Repeating the above steps, we can get a subsequence of $\left\{u_{j}^{i} \mid j=1,2, \ldots\right\}$ which we denote by $\left\{u_{j}^{i+1} \mid j=1,2, \ldots\right\}(i=1,2, \ldots)$ and satisfies the following.

$\left(1^{0}\right)$ For any fixed $i,\left\{u_{j}^{i+1}\right\}$ is a subsequence of $\left\{u_{j}^{i}\right\}$.

$\left(2^{0}\right)$ For any fixed $i, u_{j}^{i+1} \rightarrow w_{i+1}$ in $D_{i+1}($ as $j \rightarrow \infty)$, where $w_{i+1} \in W^{1, p(x)}\left(D_{i+1}\right) \cap$ $C^{1, \alpha_{i+1}}\left(D_{i+1}\right)$ satisfies $w_{i}=\left.w_{i+1}\right|_{D_{i}}$.

$\left(3^{0}\right)$ For any fixed $i, w_{i}$ satisfies

$$
\int_{D_{i}}\left|\nabla w_{i}\right|^{p(x)-2} \nabla w_{i} \nabla q d x+\int_{D_{i}} \rho(x) e^{f\left(x, w_{i}\right)} q d x=0, \quad \forall q \in W_{0}^{1, p(x)}\left(D_{i}\right) .
$$

Thus, we can conclude that

(i) $\left\{u_{j}^{j}\right\}$ is a subsequence of $\left\{u_{j}\right\}$,

(ii) there exists a function $w \in W_{\text {loc }}^{1, p(x)}(\Omega) \cap C_{\text {loc }}^{1, \alpha}(\Omega)$ such that $w_{i}=\left.w\right|_{D_{i}}$, and for any $x \in \Omega$, there exists a constant $j_{x}$ such that when $j \geq j_{x}, u_{j}^{j}(x)$ is defined at $x$, and $\lim _{j \rightarrow \infty} u_{j}^{j}(x)=w(x)$,

(iii)

$$
\int_{\Omega}|\nabla w|^{p(x)-2} \nabla w \nabla q d x+\int_{\Omega} \rho(x) e^{f(x, w)} q d x=0, \quad \forall q \in W_{0, \mathrm{loc}}^{1, p(x)}(\Omega) .
$$

Obviously, $w$ is a boundary blow-up solution for $(\mathrm{P})$.

This completes the proof. 
In Theorem 4.1, when $\inf _{x \in \Omega} p(x)>N$, the existence of solutions for $(\mathrm{P})$ is given. In the following, we will consider the existence of solutions for $(\mathrm{P})$ in the general case $1<\inf _{x \in \Omega} p(x) \leq \sup _{x \in \Omega} p(x)<\infty$. We need to do some preparation. Let us consider

$$
\begin{aligned}
\left(r^{N-1}\left|u^{\prime}\right|^{p(r)-2} u^{\prime}\right)^{\prime} & =r^{N-1} \rho(r) e^{f(r, u)}, \quad r \in\left(0, R_{\curlywedge}\right), \\
u^{\prime}(0) & =0, \quad u\left(R_{\curlywedge}\right)=d,
\end{aligned}
$$

where $R_{\curlywedge} \in(0, R)$ and $d$ is a constant.

Lemma 4.2. If $\Phi_{2}\left(R_{\curlywedge}\right) \leq d \leq \Phi_{1}\left(R_{\curlywedge}\right)$, where $\Phi_{1}$ and $\Phi_{2}$ are defined in Theorems 3.13.2, respectively, then (4.13) has a solution $u$ satisfying

$$
\Phi_{2}(r) \leq u(r) \leq \Phi_{1}(r), \quad \forall r \in\left[0, R_{\curlywedge}\right]
$$

Proof. Denote

$$
h(r, u)= \begin{cases}e^{f\left(r, \Phi_{1}(r)\right)}+\arctan \left(u(r)-\Phi_{1}(r)\right), & u(r)>\Phi_{1}(r), \\ e^{f(r, u)}, & \Phi_{2}(r) \leq u(r) \leq \Phi_{1}(r), \\ e^{f\left(r, \Phi_{2}(r)\right)}+\arctan \left(u(r)-\Phi_{2}(r)\right), & u(r)<\Phi_{2}(r) .\end{cases}
$$

Let $\rho_{E}(t)=\rho(|t|)$, and $h_{E}(t, u)=h(|t|, u)$, for all $t \in\left[-R_{\lambda}, R_{\curlywedge}\right]$. Let us consider the even solutions of the following

$$
\begin{gathered}
\left(|t|^{N-1}\left|u^{\prime}\right|^{p(t \mid)-2} u^{\prime}\right)^{\prime}=|t|^{N-1} \rho_{E}(t) h_{E}(t, u), \quad t \in\left(-R_{\curlywedge}, R_{\curlywedge}\right), \\
u\left(-R_{\curlywedge}\right)=d, \quad u\left(R_{\curlywedge}\right)=d .
\end{gathered}
$$

It is easy to see that $u$ is an even solution for (4.15) if and only if $u$ is even and

$$
u=d-\int_{r}^{R_{\lambda}}\left[|t|^{1-N} \int_{0}^{t}|s|^{N-1} \rho(s) h(s, u(s)) d s\right]^{1 /(p(t)-1)} d t, \quad \forall r \in\left[0, R_{\curlywedge}\right]
$$

Denote $\Psi(u, \mu)=\mu d-\mu \int_{r}^{R_{\lambda}}\left[|t|^{1-N} \int_{0}^{t}|s|^{N-1} \rho(s) h(s, u(s)) d s\right]^{1 /(p(t)-1)} d t$. Similar to the proof of Lemma 2.3 of [18], for any $\mu \in[0,1]$, it is easy to see that $\Psi(u, \mu)$ is compact continuous and bounded from $C_{E}^{1}\left[0, R_{\curlywedge}\right]$ to $C_{E}^{1}\left[0, R_{\curlywedge}\right]$, where $C_{E}^{1}\left[0, R_{\curlywedge}\right]=\left\{u \in C^{1}\left[0, R_{\curlywedge}\right] \mid\right.$ $u$ is even $\}$. Thus, $u=\Psi(u, 1)$ has a solution $u$ in $C_{E}^{1}\left[0, R_{\curlywedge}\right]$ and satisfies $u^{\prime}(0)=\lim _{r \rightarrow 0^{+}} u^{\prime}(r)=$ 0 . Then, $u(|t|)$ is an even solution for (4.15).

Denote $\Phi_{1, E}(t)=\Phi_{1}(|t|), \Phi_{2, E}(t)=\Phi_{2}(|t|)$. From the definitions of $\Phi_{1}$ and $\Phi_{2}$, we can see that $\Phi_{1}^{\prime}(0)=0=\Phi_{2}^{\prime}(0)$; therefore, $\Phi_{1, E}(t)$ and $\Phi_{2, E}(t)$ are supersolution and subsolution for (4.15), respectively. 
Since $\Phi_{2}\left(R_{\curlywedge}\right) \leq u\left(R_{\curlywedge}\right) \leq \Phi_{1}\left(R_{\curlywedge}\right)$ and $h_{E}(t, \cdot)$ is increasing, from the comparison principle, we have

$$
\Phi_{2, E}(t) \leq u(t) \leq \Phi_{1, E}(t), \quad \forall t \in\left[-R_{\curlywedge}, R_{\curlywedge}\right]
$$

It means that $u$ is a solution for (4.13) and $u$ satisfies

$$
\Phi_{2}(r) \leq u(r) \leq \Phi_{1}(r), \quad \forall r \in\left[0, R_{\curlywedge}\right]
$$

Thus $u$ is a radial solution for $(\mathrm{P})$. This completes the proof.

Theorem 4.3. If $f(r, u)$ satisfies

$$
f(r, u) \geq a u^{s} \quad(\text { as } u \longrightarrow+\infty) \text { for } r \in[\sigma, R) \text { uniformly, }
$$

where $\sigma$ is defined in $\left(H_{4}\right)$ and $a$ and s are positive constants, then $(\mathrm{P})$ possesses a boundary blow-up solution.

Proof. From Lemma 4.2, we have that (4.4) has a weak solution $u_{j}(x)=u_{j}(|x|)=u_{j}(r)$. Similar to the proof of Theorem 4.1, we can obtain the existence of solutions for $(\mathrm{P})$.

\section{Acknowledgments}

This paper is partly supported by the National Science Foundation of China (10701066\& 10926075 \& 10971087) China Postdoctoral Science Foundation-funded project (20090460969), and the Natural Science Foundation of Henan Education Committee (2008-755-65).

\section{References}

[1] Y. Chen, S. Levine, and M. Rao, "Variable exponent, linear growth functionals in image restoration," SIAM Journal on Applied Mathematics, vol. 66, no. 4, pp. 1383-1406, 2006.

[2] M. Růžička, Electrorheological Fluids: Modeling and Mathematical Theory, vol. 1748 of Lecture Notes in Mathematics, Springer, Berlin, Germany, 2000.

[3] V. V. Zhikov, "Averaging of functionals of the calculus of variations and elasticity theory," Mathematics of the USSR-Izvestiya, vol. 29, pp. 33-36, 1987.

[4] E. Acerbi and G. Mingione, "Regularity results for a class of functionals with non-standard growth," Archive for Rational Mechanics and Analysis, vol. 156, no. 2, pp. 121-140, 2001.

[5] A. Coscia and G. Mingione, "Hölder continuity of the gradient of $p(x)$-harmonic mappings," Comptes Rendus de l'Académie des Sciences. Série I. Mathématique, vol. 328, no. 4, pp. 363-368, 1999.

[6] X. Fan and D. Zhao, "On the spaces $L^{p(x)}(\Omega)$ and $W^{m, p(x)}(\Omega)$," Journal of Mathematical Analysis and Applications, vol. 263, no. 2, pp. 424-446, 2001.

[7] X.-L. Fan, H.-Q. Wu, and F. Wang, "Hartman-type results for $p(t)$-Laplacian systems," Nonlinear Analysis: Theory, Methods \& Applications, vol. 52, no. 2, pp. 585-594, 2003.

[8] X. Fan, "Global $C^{1, \alpha}$ regularity for variable exponent elliptic equations in divergence form," Journal of Differential Equations, vol. 235, no. 2, pp. 397-417, 2007.

[9] X.-L. Fan and Q.-H. Zhang, "Existence of solutions for $p(x)$-Laplacian Dirichlet problem," Nonlinear Analysis: Theory, Methods \& Applications, vol. 52, no. 8, pp. 1843-1852, 2003.

[10] X. Fan, Q. Zhang, and D. Zhao, "Eigenvalues of $p(x)$-Laplacian Dirichlet problem," Journal of Mathematical Analysis and Applications, vol. 302, no. 2, pp. 306-317, 2005. 
[11] O. Kováčik and J. Rákosník, “On spaces $L^{p(x)}(\Omega)$ and $W^{k, p(x)}(\Omega)$, , Czechoslovak Mathematical Journal, vol. 41(116), no. 4, pp. 592-618, 1991.

[12] P. Marcellini, "Regularity and existence of solutions of elliptic equations with $p, q$-growth conditions," Journal of Differential Equations, vol. 90, no. 1, pp. 1-30, 1991.

[13] S. G. Samko, "Density $C_{0}^{\infty}\left(\mathbb{R}^{N}\right)$ in the generalized Sobolev spaces $W^{m, p(x)}\left(\mathbb{R}^{N}\right)$," Doklady Rossiuskaya Akademii Nauk, vol. 369, no. 4, pp. 451-454, 1999.

[14] Q. Zhang, "A strong maximum principle for differential equations with nonstandard $p(x)$-growth conditions," Journal of Mathematical Analysis and Applications, vol. 312, no. 1, pp. 24-32, 2005.

[15] Q. Zhang, Z. Qiu, and R. Dong, "Existence and asymptotic behavior of positive solutions for a variable exponent elliptic system without variational structure," Nonlinear Analysis: Theory, Methods $\mathcal{E}$ Applications, vol. 72, no. 1, pp. 354-363, 2010.

[16] Q. Zhang, "Existence and asymptotic behavior of blow-up solutions to a class of $p(x)$-Laplacian problems," Journal of Mathematical Analysis and Applications, vol. 329, no. 1, pp. 472-482, 2007.

[17] Q. Zhang, "Existence and asymptotic behavior of positive solutions to $p(x)$-Laplacian equations with singular nonlinearities," Journal of Inequalities and Applications, vol. 2007, Article ID 19349, 9 pages, 2007.

[18] Q. Zhang, "Existence of solutions for weighted $p(r)$-Laplacian system boundary value problems," Journal of Mathematical Analysis and Applications, vol. 327, no. 1, pp. 127-141, 2007.

[19] L. Bieberbach, " $\Delta u=e^{u}$ und die automorphen Funktionen," Mathematische Annalen, vol. 77, no. 2, pp. 173-212, 1916.

[20] C. Anedda, A. Buttu, and G. Porru, "Second-order estimates for boundary blowup solutions of special elliptic equations," Boundary Value Problems, vol. 2006, Article ID 45859, 12 pages, 2006.

[21] S. Kichenassamy, "Boundary blow-up and degenerate equations," Journal of Functional Analysis, vol. 215, no. 2, pp. 271-289, 2004.

[22] J. B. Keller, "On solutions of $\Delta u=f(u)$," Communications on Pure and Applied Mathematics, vol. 10, pp. 503-510, 1957.

[23] A. V. Lair, "A necessary and sufficient condition for existence of large solutions to semilinear elliptic equations," Journal of Mathematical Analysis and Applications, vol. 240, no. 1, pp. 205-218, 1999.

[24] A. Mohammed, "Existence and asymptotic behavior of blow-up solutions to weighted quasilinear equations," Journal of Mathematical Analysis and Applications, vol. 298, no. 2, pp. 621-637, 2004.

[25] J. Matero, "Quasilinear elliptic equations with boundary blow-up," Journal d'Analyse Mathématique, vol. 69, pp. 229-247, 1996.

[26] R. Osserman, “On the inequality $\Delta u \geq f(u)$," Pacific Journal of Mathematics, vol. 7, pp. 1641-1647, 1957. 


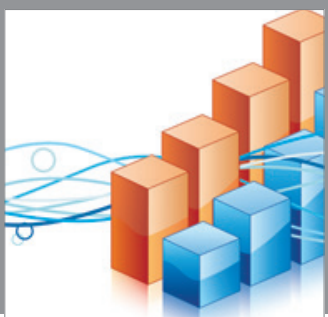

Advances in

Operations Research

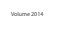

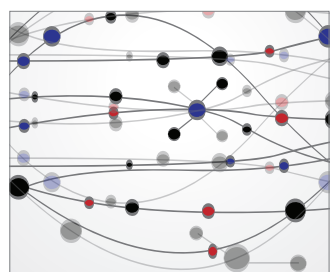

\section{The Scientific} World Journal
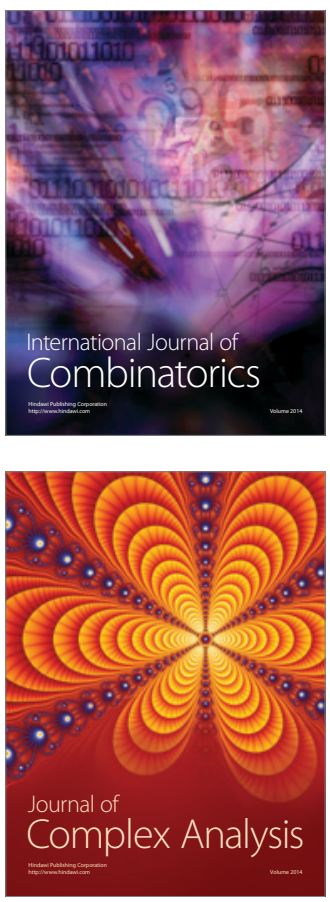

International Journal of

Mathematics and

Mathematical

Sciences
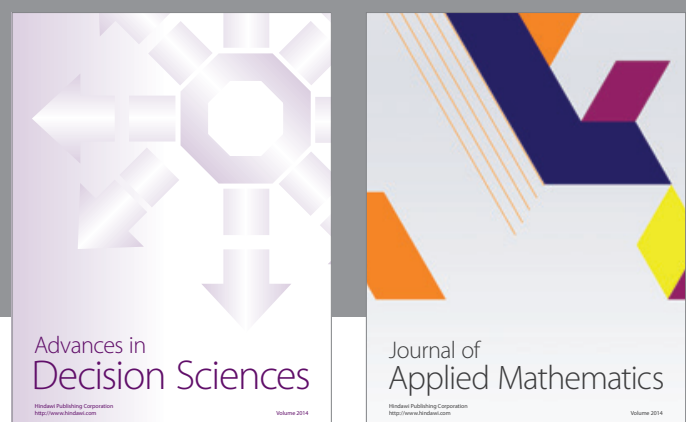

Journal of

Applied Mathematics
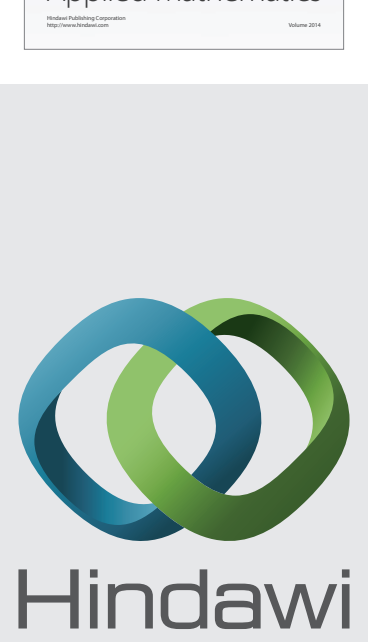

Submit your manuscripts at http://www.hindawi.com
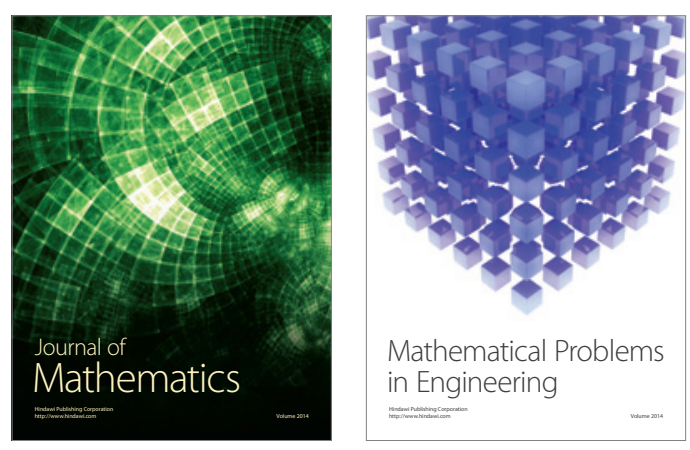

Mathematical Problems in Engineering
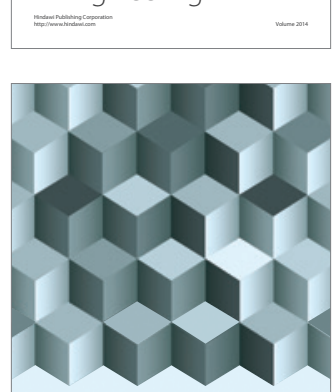

Journal of

Function Spaces
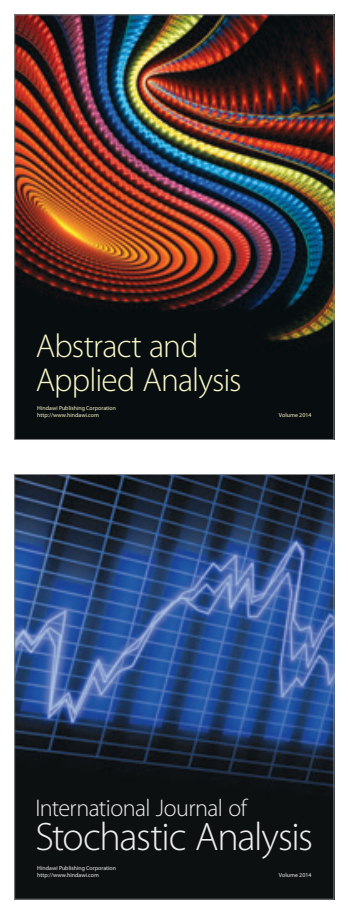

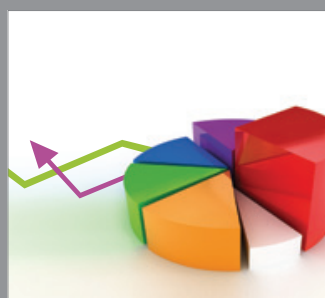

ournal of

Probability and Statistics

Promensencen
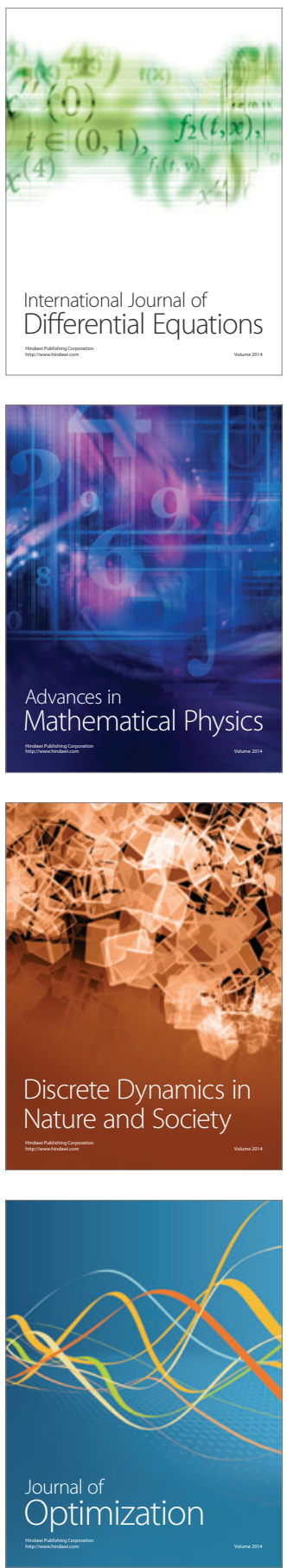